\title{
Cardiac safety implications of hNav1.5 blockade and a framework for pre-clinical evaluation
}

\author{
Gül Erdemli ${ }^{1,2 *}$, Albert M. Kim ${ }^{2,3}$, Haisong Ju ${ }^{2,4}$, Clayton Springer ${ }^{5}$, Robert C. Penland ${ }^{6}$ and \\ Peter K. Hoffmann ${ }^{2,4}$
}

${ }^{1}$ Center for Proteomic Chemistry, Novartis Institutes for Biomedical Research, Cambridge, MA, USA

${ }^{2}$ Translational Cardiovascular Advisory Team at Novartis, Cambridge, MA, USA

${ }^{3}$ Translational Medicine, Novartis Institutes for Biomedical Research, Cambridge, MA, USA

${ }^{4}$ Preclinical Safety, Novartis Institutes for Biomedical Research, East Hanover, NJ, USA

${ }^{5}$ Global Discovery Chemistry, Novartis Institutes for Biomedical Research, Cambridge, MA, USA

${ }^{6}$ Modeling and Simulation, Global Development, Novartis Pharmaceuticals Corporation, Cambridge, MA, USA

\author{
Edited by: \\ Jufeng Wang, Waylandgreen, USA \\ Reviewed by: \\ Sirajudheen Anwar, University of \\ Messina, Italy \\ Jufeng Wang, Waylandgreen, USA \\ *Correspondence: \\ Gül Erdemli, Novartis Institutes for \\ Biomedical Research, 250 \\ Massachusetts Avenue, Cambridge, \\ MA 02139, USA. \\ e-mail: gul.erdemli@novartis.com
}

\begin{abstract}
The human cardiac sodium channel (hNav1.5, encoded by the SCN5A gene) is critical for action potential generation and propagation in the heart. Drug-induced sodium channel inhibition decreases the rate of cardiomyocyte depolarization and consequently conduction velocity and can have serious implications for cardiac safety. Genetic mutations in hNav1.5 have also been linked to a number of cardiac diseases. Therefore, off-target hNav1.5 inhibition may be considered a risk marker for a drug candidate. Given the potential safety implications for patients and the costs of late stage drug development, detection, and mitigation of hNav1.5 liabilities early in drug discovery and development becomes important. In this review, we describe a pre-clinical strategy to identify hNav1.5 liabilities that incorporates in vitro, in vivo, and in silico techniques and the application of this information in the integrated risk assessment at different stages of drug discovery and development.
\end{abstract}

Keywords: sodium channel, arrhythmia, pre-clinical, ECG

\section{INTRODUCTION}

Cardiovascular safety is a principal concern in drug development because undesired drug effects can have serious consequences for cardiovascular physiology, impacting vital processes such as heart rhythm, contractility, cardiac output, blood pressure, and end organ perfusion. Indeed, cardiovascular safety is the leading cause of post market drug withdrawal in the US (Piccini et al., 2009). A drug candidate's proarrhythmic risk is thus of significant concern throughout drug development. Much of this concern is based on experience over the past several decades with compounds that block the human ether-à-go-go related gene (hERG) potassium channel. Abundant pre-clinical and clinical observations have established a clear relationship between hERG blockers, a risk for drug-induced QT prolongation, and Torsades de Pointes (TdP), a potentially lethal cardiac arrhythmia (Heist and Ruskin, 2010; Van Noord et al., 2010). It is also recognized that drug-induced effects at other cardiac ion channels can result in serious adverse cardiovascular effects (Chen et al., 2009). This has spurred pharmaceutical companies to implement routine screening of drug candidates against a panel of cardiac ion channels early in drug development, and there is an increasing need in understanding how results of these pre-clinical screens translate into clinical safety risks in patients (Cao et al., 2010; Gintant et al., 2011; Harmer et al., 2011).

The cardiac sodium channel (hNav1.5, encoded by the SCN5A gene) is responsible for electrical excitation of atrial and ventricular myocytes. Voltage-dependent opening of the channel drives the upstroke of the cardiac action potential (phase 0 ), which initiates cellular depolarization and propagates the action potential throughout the cardiac chambers (George et al., 1995; Balser, 2001; Keating and Sanguinetti, 2001). Sodium channel inhibition decreases the rate of cardiac depolarization and consequently slows conduction velocity. Depending on the magnitude, hNav1.5 blockade may manifest as PR interval prolongation and/or QRS complex widening on the electrocardiogram (ECG; Vaughan Williams, 1992; Bode-Schnurbus et al., 2003; Hummel et al., 2009; Gintant et al., 2011).

It is important to note that drug related effects on sodium channels can also modify cardiac repolarization. For example, decreased inactivation of the sodium inward current can prolong the action potential (Balser, 2001). Also, inhibition of cardiac sodium channels can mitigate hERG channel blocking effects of drugs as shown for the antipsychotic compound clozapine (Warner and Hoffmann, 2002). Furthermore, sodium channel inhibition can lead to impairment of cardiac contractility due to reduced cytosolic free calcium concentrations mediated by the $\mathrm{Na}^{+} / \mathrm{Ca}^{2+}$ exchanger (Ito et al., 1996; Larbig et al., 2010).

In general, sodium channels are susceptible to blockade by a wide variety of small molecules, some of which are used clinically as local anesthetics, anticonvulsants, antidepressants, and antiarrhythmics (Freedman and Steinberg, 1991; Graf, 2001; Thanacoody and Thomas, 2005). Many of these drugs are associated with serious adverse reactions including cardiac arrhythmias (Vieweg and Wood, 2004).

A considerable body of data indicates that drug induced or genetic alterations of the cardiac sodium channel can have important cardiovascular consequences. Genetic mutations in the SCN5A gene underlie a number of cardiac diseases with diverse 
phenotypes (Tfelt-Hansen et al., 2010). Congenital loss and gain of function mutations in SCN5A can both increase arrhythmia risk. For example, loss of function mutations in SCN5A cause Brugada syndrome while gain of function mutations cause long QT syndrome type 3. Moreover, a striking lesson learned from pharmacologically induced hNav1.5 inhibition came in the form of the cardiac arrhythmia suppression trial (CAST), which tested the hypothesis that suppressing ventricular premature complexes in patients after myocardial infarction would improve survival. Rather than the expected benefit, CAST treatment arms using encainide or flecainide were associated with increased mortality due to arrhythmias (Echt et al., 1991).

Off-target hNav1.5 channel inhibition is considered a risk marker for a drug candidate. However, some uncertainty remains regarding the implications of QRS widening and its translation to pro-arrhythmic risk. While having a wide QRS appears to confer arrhythmic risk in certain target patient populations with left (Sumner et al., 2009) or right bundle branch block (Adesanya et al., 2008), the relationship between QRS interval prolongation and arrhythmias in patients without underlying heart disease is less clear (Seger, 2006).

Given the weight of evidence regarding the adverse safety risks and impact of cardiac sodium channel inhibition, detection, and mitigation of hNav1.5 blockade is an important shared goal in the pharmaceutical industry. In this review, we describe our current pre-clinical strategy incorporating in vitro, in vivo, and in silico techniques to identify the potential of new drug candidates for hNav1.5 blockade and its electrophysiological consequences. We also discuss examples of how to apply these results in an integrated risk assessment at different stages of drug discovery.

\section{REGULATORY GUIDANCE}

The two relevant regulatory documents that provide guidance for investigating drug effects on cardiac ion channels are International Conference on Harmonization (ICH) guidance for industry: S7A Safety pharmacological studies for human pharmaceuticals (ICH S7A) and S7B Non-clinical Evaluation of the Potential for Delayed Ventricular Repolarization by Human Pharmaceuticals $\left(\mathrm{ICH} \mathrm{S7B} \mathrm{B}^{1}\right)$. But, in contrast to regulatory precedents regarding the rapid delayed rectifier potassium current $\left(\mathrm{I}_{\mathrm{Kr}}\right)$ and its relationship to the electrocardiographic QT interval, evaluation of effects on the cardiac sodium channel on changes in electrocardiographic $\mathrm{PR}$, and QRS intervals are not addressed in detail. ICH S7A states that effects of the test substance on the ECG including effects on conduction abnormalities should be evaluated.

ICH S7B describes the role of sodium channels during phase 0 and phase 1 of the action potential. Although testing for effects on the cardiac sodium channel is not mentioned explicitly in the primary testing strategy, this issue is addressed in the Follow up Studies section, where the potential for inhibition of non-hERG cardiac ion channels and their possible role in mitigating $\mathrm{I}_{\mathrm{Kr}}$ effects on action potential duration (APD) and QT interval are mentioned. For in vivo experiments, measurements of PR interval and QRS duration are recommended in ICH S7B.

\footnotetext{
${ }^{1}$ http://www.ich.org/products/guidelines/safety/article/safety-guidelines.html
}

\section{PRE-CLINICAL ASSESSMENT IN VITRO ASSAYS AND IN SILICO TOOLS Patch-clamp}

A common initial step in pre-clinical cardiac safety evaluation is the in vitro assessment of effects of compounds on ion channel activity in recombinant expression systems using patch-clamp electrophysiology. Conventional manual patch-clamp from single cells with GigaOhm seals is the gold standard technology. However, the throughput of conventional electrophysiology assays is too low to meet drug discovery project needs and requires highly trained personnel. Recent developments in automated patchclamp technologies have enabled high throughput profiling of compounds during early stages of drug discovery (Dunlop et al., 2008; Harmer et al., 2008; Castle et al., 2009; Chen et al., 2009; Cao et al., 2010). Biophysical properties and pharmacological profiles obtained from automated hNav1.5 assays show good concordance with manual patch-clamp recordings (Harmer et al., 2008; Castle et al., 2009; Cao et al., 2010). Because many clinically used sodium channel blockers are state-dependent modulators, evaluation of state dependence is an important part of pre-clinical safety risk assessment (Cao et al., 2010). Automated patch-clamp assays have also been shown to recognize state-dependent modulators for the hNav1.5 channel (Harmer et al., 2008; Castle et al., 2009; Cao et al., 2010). While many competing technologies are available, Novartis has utilized the IonWorks Quattro ${ }^{\mathrm{TM}}$ system (Molecular Devices, part of MDS Analytical Technologies) for hNav1.5 profiling for several years (Cao et al., 2010). The assay is used routinely as part of an integrated pre-clinical cardiac ion channel safety assessment during phase transitions such as lead nomination and clinical candidate selection. Lead candidate profiling has allowed prioritization of chemical series based on effects on cardiac ion channels, including hNav1.5, and subsequently the informed selection of compounds without significant ion channel safety liabilities for further development. When hNav1.5 activity is identified within a chemical series, the assay is used to investigate structure activity relationships (SAR) and to guide medicinal chemistry efforts for mitigation of hNav1.5 channel inhibition during lead optimization.

Several limitations of automated patch-clamp systems should be kept in mind. Physicochemical properties of compounds such as hydrophobicity and solubility can limit measurement of the true inhibitory potency. Particularly for the IonWorks Quattro system, the lack of a fluid outlet and other technical limitations confines this system to single time point readings making it unsuitable for kinetic profiling (Cao et al., 2010). To study inhibition kinetics and reversibility, additional manual patch-clamp assays may be required. In this context, manual patch-clamp experiments complement the data generated from automated experiments, which helps define a precise channel-compound interaction, allows measuring actual concentrations of the test compound in the recording chamber and recordings at physiological temperatures.

While factors that directly modulate ionic channel proteins can be explored using patch-clamp assays, indirect modulation through mechanisms like gene expression or protein trafficking requires different techniques. Alterations in the hNav1.5 expression levels have been associated with acquired cardiac disorders, such as heart failure (Rook et al., 2012). Cardiac sodium channel 
blockers have also been shown to facilitate trafficking of mutant hNav1.5 proteins in patients with Long QT Syndrome type 3 (Ruan et al., 2010). However, to our knowledge there are no reports of small molecule inhibitors of hNav1.5 that act by altering channel trafficking and thereby cause delayed ECG changes in conduction in vivo.

\section{In silico tools and structure activity trends in the patch-clamp data}

Since implementation of the automated patch-clamp hNav1.5 assay at Novartis, 1945 compounds across a range of different targets have been tested and 148 of these showed inhibition of the hNav1.5 channel $\left(\mathrm{IC}_{50}<10 \mu \mathrm{M}\right.$ ). Using ICM (version 3.7-2c, MolSoft. LLC), these compounds were clustered (at the 0.2 Tanimoto level) into 895 clusters. Of the 895 clusters, 90 clusters have at least 1 member that is a hNav1.5 inhibitor $\left(\mathrm{IC}_{50}<10 \mu \mathrm{M}\right)$. To analyze the SAR, RDKit's radius $=3$ Morgan count fingerprints hashed into a vector of size 1024 (RDKit: Open-source cheminformatics $^{2}$ ) was used. The data set was analyzed for the effects of small, lead optimization-sized changes which were defined as a Manhattan distance of 70 or less in the Morgan count fingerprints. Because each individual observed change is not statistically significant, a quantitative structure activity relationship (QSAR) model was constructed to help identify trends. Figure 1 shows three selected chemical changes that had a dramatic effect on hNav1.5 blockade potency and follow the trends observed in the data. The first observed change (Figure 1A) was the replacement of the aliphatic cyclopentyl chain with aliphatic chain with hydroxyls. The replacement of the carboxylic acid benzothiazole with another aryl system (many of the examples have a heteroatom closer to the acid group) reduced the hNav1.5 inhibition (Figure 1B). The

${ }^{2}$ http://www.rdkit.org

A

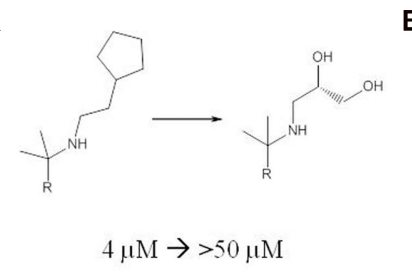

C

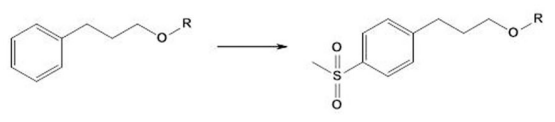

$3 \mu \mathrm{M} \rightarrow>50 \mu \mathrm{M}$ addition of a sulfone to a phenyl group which itself is at the end of aliphatic tail (Figure 1C) reduced hNav1.5 blockade.

With only 1945 compounds measured to date, it is still early in the exploration of hNav1.5 blockade and there is much more SAR left to be discovered. Although hNav1.5 SAR is overall much less promiscuous than the SAR for the hERG channel, some of approaches that are useful for mitigation of hERG also apply (Jamieson et al., 2006; Papadatos et al., 2010). For example, reducing the $\log \mathrm{P}$ of compounds, and adding hydroxyls to greasy motifs reduce hNav1.5 blockade.

\section{Cardiac tissue and isolated heart preparations}

Building on the profiling established through automated or manual patch-clamp assays, cardiac sodium channel blocking effects of drug candidates can be further evaluated in vitro using cardiac tissues and isolated heart preparations. Acute pharmacologic effects can be assessed by measuring QRS interval from the ECG in Langendorff perfused hearts and arterially perfused left ventricular wedge preparations (Lu et al., 2010). Hondeghem et al. (2003) demonstrated that the SCREENIT model (rabbit heart) could properly characterize prototypical class IA, IB and IC sodium channel blockers (e.g., quinidine, lidocaine, and flecainide). Lu et al. (2010) showed that these models can differentiate the proarrhythmic effects of sodium channel blockers that affect cardiac conduction. For example, in isolated left ventricular wedge preparations, flecainide increased QRS interval in a rate-dependent manner, and elicited ventricular tachycardia. On the other hand, lidocaine only slightly increased QRS duration without inducing ventricular tachycardia (Lu et al., 2010). Similarly in isolated rabbit hearts, flecainide was associated with a marked increase in QRS interval and ventricular tachycardia, whereas, lidocaine produced a small increase in QRS duration and did not induce ventricular tachycardia (Lu et al., 2010). In addition to providing data about Nav1.5 liabilities, the isolated heart and wedge preparations are also useful to evaluate compounds that have effects on multiple cardiac ion channels and pro-arrhythmia potential.

While the rabbit ventricular wedge and isolated rabbit heart models are useful for assessing Nav 1.5 dependent electrophysiologic effects of compounds in early drug discovery, these models have limitations as well. These assays have low throughput and require highly trained individuals. The results are subject to discrepancies comparable to other in vitro and in vivo models due to species specific physiology and pharmacology, differences in assay conditions (e.g., perfusion time, recording frequencies, and temperature) and residual effects of anesthetics (Lu et al., 2007; Chen et al., 2009). Such models may also require relatively large quantities of compounds if high concentrations need to be tested, limiting their routine use in the very early stages of drug discovery. At Novartis, we use the isolated rabbit heart model on a case-bycase basis for mechanistic studies when there are discrepancies between in vitro and in vivo results or if there are pro-arrhythmia concerns.

\section{IN VIVO ANIMAL MODELS}

Cardiac sodium channel liability should be further evaluated in vivo using conscious and in some cases anesthetized animal models. In conscious animal models, Nav1.5 blocking risk is 
routinely assessed by evaluating QRS and PR intervals on the ECG. The ECG can be recorded continuously in conscious, non-restrained dogs or monkeys with an implanted telemetry transmitter or using an external jacket (non-invasive telemetry). In anesthetized models, intracardiac recordings like the His bundle electrogram (HBE) can be obtained and provide valuable functional information. However, several caveats must be considered for anesthetized animals. Since conditions are only stable for a few hours, such experiments provide only a limited time window to evaluate a drug effect. Thus, Nav1.5 blocking effects due to metabolites or myocardial accumulation of the parent compound may be missed. In addition, some general anesthetics such as pentobarbital can affect cardiac conduction at anesthetic concentrations and may confound results. A recent study evaluated the value of several in vivo pre-clinical models to predict the cardiac sodium blocking risk in human (Heath et al., 2011). Translation of flecainide- or mexiletine-induced cardiac sodium channel inhibition and slowing of ventricular conduction from pre-clinical models to the clinic was assessed using conscious telemetered rat, dog, and anesthetized dog models. Effects of flecainide and mexiletine in conscious dogs were comparable to the ECG effects observed in patients and healthy volunteers. The anesthetized dog was least sensitive for detecting QRS changes. This study concluded that the conscious dog was the most predictive model for detecting effects of compounds on cardiac conduction as compared to conscious rat telemetry or anesthetized dog models.

At Novartis, we routinely use external jacket telemetry in conscious dogs or monkeys in the first non-rodent toxicity studies to detect compound related ECG changes that may be due to blockade of Nav1.5. We may also employ invasive telemetry in conscious dogs or monkeys at this early stage to better characterize equivocal or relatively small ECG effects observed in previous studies. For some compounds, the first in vivo evidence of sodium channel blocking effect at low drug exposures may be $\mathrm{PR}$ interval prolongation without QRS prolongation (see compounds NVP-3, NVP-4, and NVP-6 in Table 1). For the $\mathrm{PR}$ interval it is important to differentiate between a sodium channel blocker and a calcium channel blocker. This can be achieved by testing the activity of compounds on the cardiac calcium channel (hCav1.2) in vitro as well as recording intracardiac conduction intervals in anesthetized dogs. The AH and HV intervals are measured (Inoue and Zipes, 1987) to further assess drug effects on the cardiac conduction system. The $\mathrm{AH}$ interval is measured from the earliest onset of rapid right atrial activity to the onset of the His potential and largely represents conduction time through the atrioventricular (AV) node. The HV interval is measured from the onset of the His bundle deflection to the beginning of ventricular depolarization and represents infra Hisian conduction from below the AV node to ventricular myocardium. Cardiac sodium channel blockers are associated with QRS and PR interval prolongation on the ECG and $\mathrm{HV}$ interval prolongation; in contrast, typical findings for calcium channel blockers include PR prolongation on the ECG and AH interval prolongation (Pressler et al., 1995). In addition, calcium channel blockers often lower blood pressure and cause AV block at high doses, observations that are uncommon for Nav1.5 blockers (Takahara et al., 2001). While several disease models (in vitro and in vivo torsade de pointes models) have been developed to assess the pro-arrhythmic/torsadogenic potential of compounds that inhibit $\mathrm{I}_{\mathrm{Kr}}$ and prolong the QT interval, analogous models have not been validated for compounds that inhibit cardiac sodium channel and result in the PR and/or QRS prolongation.

\section{MODELING AND SIMULATION}

Mathematical models were first introduced to describe the nerve action potential in the squid giant axon (Hodgkin and Huxley, 1952). Subsequently, they have evolved to describe electrical excitation of cardiac tissues (for a historical perspective see Noble and Rudy, 2001), and have provided mechanistic insight into how pharmacologically or genetically mediated perturbations affect electrophysiology from the single ion channel level to the whole heart. Current cellular mathematical models describe the electrical activity of a single cell by relating the transmembrane potential difference to the function of different ion channels (e.g., sodium flux through the hNav1.5 channel), exchangers (e.g., $\mathrm{Na}^{+} / \mathrm{Ca}^{2+}$ ) and electrogenic pumps (e.g., $\mathrm{Na}^{+} / \mathrm{K}^{+}$ATPase). The sodium channel current has been modeled extensively under normal physiological conditions and in disease states (Moreno et al., 2011). Advances in molecular biology have enabled researchers to isolate, build, and analyze models for congenital defects in channel proteins (Clancy and Rudy, 2002; Starmer et al., 2003a) that complement models of pharmacological drug effects (Starmer et al., 2003b).

Early models treated cardiac sodium channel inhibition as a simple reduction in conductance. However, more recent models incorporate components that address more complex statedependent ligand-channel interactions that are crucial to explaining the observed behavior of certain drugs. A good example of how modeling has provided translational insight into how sodium channel inhibition can result in life-threatening ventricular arrhythmias was provided in analyzing the results from the CAST clinical trial. Modeling studies suggested clear links between cardiac sodium channel inhibition, the arrhythmogenic vulnerable period, and increase in ectopic activity (Starmer et al., 1991, 1992). These and other studies (Starmer et al., 2003b) indicate that hNav1.5 inhibition with a small molecule can induce pro-arrhythmia, particularly in patients with underlying ischemic heart disease.

Mathematical models have been useful in predicting the complex effects of drugs which inhibit multiple cardiac ion channels. For example, a model was developed to quantitatively predict the degree of sodium channel blockade required to offset the effects of a hERG channel blocker on action potential prolongation in canine Purkinje fibers (Bottino et al., 2006). The estimated $\mathrm{IC}_{50}$ values for hNav1.5 inhibition were subsequently confirmed with data from independent patch-clamp experiments (Bottino et al., 2006). Novartis use models in conjunction with patch-clamp data from hERG, hNav1.5 and hCav1.2 channels to then conduct virtual action potential and tissue conduction simulations to determine the integrated concentration response in advance of or in parallel with in vitro and in vivo profiling experiments on a case-by-case basis. 
Table 1 | Pre-clinical profiles of compounds with cardiac sodium channel liability.

\begin{tabular}{|c|c|c|c|c|}
\hline & \multirow[t]{2}{*}{ hNav1.5 ${ }^{1}\left(\mathrm{IC}_{50}, \mu \mathrm{M}\right)$} & \multirow[t]{2}{*}{ Isolated heart ${ }^{2,3}$ (conduction @ $\mu \mathrm{M}$ ) } & \multicolumn{2}{|c|}{ In vivo telemetry ${ }^{3,4}$} \\
\hline & & & 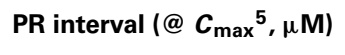 & QRS duration $\left(@ C_{\max }{ }^{5}, \mu \mathrm{M}\right)$ \\
\hline NVP-2 & $4.5^{\mathrm{M}}$ & NT & $\uparrow(4.8)$ & $\uparrow(4.8)$ \\
\hline NVP-3 & $3.5^{\mathrm{Q}}$ & NT & $\uparrow(0.07)$ & NE (1.9) \\
\hline NVP-4 & $16^{\mathrm{M}}$ & $\downarrow(9)$ & $\uparrow(1.2)$ & $\uparrow(1.9)$ \\
\hline NVP-6 & $>100^{\mathrm{M}, 6}$ & $N E(30)$ & $\uparrow(4.5)$ & $\uparrow(13.7)$ \\
\hline NVP-7 & $41^{\mathrm{Q}}$ & $\downarrow(90)$ & $\uparrow(0.14)$ & $\uparrow(0.14)$ \\
\hline NVP-8 & $366^{\mathrm{M}, 7}$ & $\downarrow(900)$ & NE (43) & NE (43) \\
\hline NVP-9 & $>30^{0,8}$ & NT & $\uparrow(1.2)$ & $\uparrow(1.2)$ \\
\hline NVP-10 & $>300^{\mathrm{M}, 9}$ & NT & $\uparrow(20.6)$ & $\uparrow(20.6)$ \\
\hline
\end{tabular}

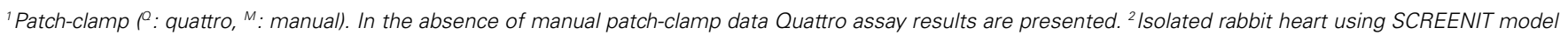
(Hondeghem, 1994). ${ }^{3}$ All animal studies have been approved by the Novartis Animal Care and Use Committee. ${ }^{4}$ Non-rodent telemetry (dog or non-human primate).

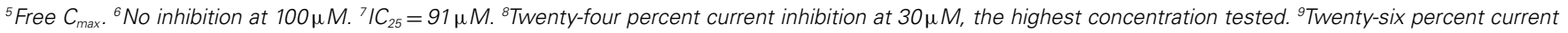
inhibition at $300 \mu \mathrm{M}$, the highest concentration tested. NT, not tested; NE, no effect.

\section{CLINICAL EVALUATION OF PHARMACOLOGICALLY MEDIATED EFFECTS ON hNAV1.5}

Because the clinical manifestations of inhibiting cardiac sodium channel can be quite variable, clinical assessment of such molecules should be approached carefully. ECG or Holter telemetry assessments are fundamental tools, and changes such as PR prolongation or QRS widening can give indications of cardiac sodium channel inhibition. A catheter-based clinical electrophysiology study may also be performed when a sodium channel blocker may be expected to elicit changes similar to those reported in non-rodent studies: prolongation of PR and/or QRS on the 12 lead ECG, and prolongation of the $\mathrm{AH}$ and/or $\mathrm{HV}$ intracardiac intervals. While studies have established a precedent for clinical evaluation of sodium channel blockers using invasive catheter studies (Heath et al., 2011), only limited data exist and do not clearly address when an invasive approach is warranted. Similarly, there are limited data available regarding the relative sensitivity and specificity of the PR, QRS, AH, and HV intervals when applied as biomarkers for hNav1.5 blockade. Ultimately, clinical assessment strategies should not only focus on standard measurements like the PR and QRS intervals, but also consider relevant factors including pharmacokinetics, safety margins, comorbidities, and concomitant medications. In addition, the strategy should be tailored to the specific development program and the target population accounting for the relative risks and benefits of invasive procedures like catheter electrophysiology studies in humans.

\section{RECENT NOVARTIS EXPERIENCE WITH THE PRE-CLINICAL ASSESSMENT OF hNAV1.5 AND CLINICAL TRANSLATION}

We have applied the pre-clinical testing strategies described above over the past several years, and exemplary results are summarized in Table 1. Five of ten Novartis compounds that were tested in vitro in the hNav1.5 patch-clamp assay, were also tested in the isolated rabbit heart model. Four of these 5 compounds showed conduction slowing, indicative of sodium channel inhibition. NVP-6, which was inactive in the patch-clamp assay (no inhibition of the
hNav1.5 current at $100 \mu \mathrm{M})$ also failed to induce any significant conduction effects in the isolated heart model at $30 \mu \mathrm{M}$ (the highest concentration tested). Overall the data show good correlation between the patch-clamp and the isolated heart assays.

All 10 compounds were studied using the in vivo non-rodent telemetry model; of these 10 compounds, 9 induced PR interval prolongation and/or QRS widening (Table 1). One compound, NVP-8, did not produce any significant effect on the ECG, with the caveat that the in vivo exposure achieved was limited. In contrast, NVP-6 and NVP-10 induced ECG changes in vivo, but did not produce a high degree of hNav1.5 inhibition in vitro. The reasons for this discrepancy are not known, but potential explanations include species differences, metabolites that may be more potent hNav1.5 inhibitors, in vivo faster heart rate and accumulation of the compound in cardiac tissue in vivo. It is also possible that the differences between the recombinant and native channel may contribute to this discrepancy. The patch-clamp hNav1.5 assay utilizes human embryonic kidney cells (HEK293) that overexpress the pore forming alpha subunit of the human cardiac sodium channel. This construct notably lacks the $\beta$ subunits and other associated proteins, which are known to regulate activity of hNav1.5 (Abriel, 2007). Though there are no known small molecule examples, it is plausible that compounds may act thorough ancillary proteins. Such effects may be underestimated or missed entirely in the patch-clamp assay, but would be revealed in vivo. This scenario underscores the importance of integrating both in vitro and in vivo assays for the pre-clinical assessment of sodium channel liability. Overall, these findings suggest that in vitro assays correlate reasonably well with in vivo non-rodent telemetry model results, but also draw attention to circumstances where assays may be insensitive or provide disparate results.

Although our longitudinal experience is limited to date, the case of NVP-4 illustrates how pre-clinical findings can translate into the clinical realm. NVP-4 had no significant effect on hERG (at $30 \mu \mathrm{M}$ ) or on hCav1.2 (at $30 \mu \mathrm{M}$ ) currents, but blocked the hNav1.5 channels with an $\mathrm{IC}_{50}$ of $15.8 \mu \mathrm{M}$ in the patch-clamp 
assay. In the rabbit isolated heart model, NVP-4 was tested at concentrations ranging between 0.3 and $30 \mu \mathrm{M}$. At concentrations $\geq 9 \mu \mathrm{M}$, conduction velocity decreased and ventricular tachycardia were observed (Table 1). In a single dose safety pharmacology dog telemetry study, dose dependent PR prolongation and QRS widening were observed without significant changes in QT interval duration (Figure 2). A 6\% PR prolongation was observed at a free $C_{\max }$ of $1.2 \mu \mathrm{M}$, and a $28 \%$ PR prolongation accompanied by a $23 \%$ QRS widening at a free $C_{\max }$ of $1.9 \mu \mathrm{M}$ (Table 1). The Nav1.5 inhibition (PR and/or QRS prolongation) was also evident in a 4 week general toxicity study in dogs at a free $C_{\max }$ range of 1.1 to $2.2 \mu \mathrm{M}$. During a 13 week general toxicity dog study, sudden deaths and polymorphic ventricular tachycardia were observed at a free $C_{\max }$ of $2.3 \mu \mathrm{M}$, with PR prolongation as the earliest sign of impaired cardiac conduction. In healthy volunteers, single doses of NVP-4 resulted in a mean $16 \mathrm{~ms}$ PR prolongation that coincided with $T_{\max }$. At this dose, the free $C_{\max }$ was $1.2 \mu \mathrm{M}$. As a result of the pre-clinical and clinical findings and a narrow safety margin, further development of NVP-4 was discontinued. In this example, both in vitro assays and dog telemetry data were consistent with and predictive of the clinical findings in healthy subjects (Figure 2).

\section{INTEGRATED ASSESSMENT STRATEGY}

A schematic of how Novartis currently applies an integrated, stepwise approach to cardiac sodium channel testing for small molecules during early drug development is outlined in Figure 3. The first step is routine in vitro assessment of ion channel activity based on automated patch-clamp electrophysiology studies in recombinant expression systems. Automated electrophysiology platforms enable higher throughput for large-scale screening compared with manual patch-clamp and therefore, can be utilized in an early phase of drug discovery such as in lead finding/lead optimization. If hNav1.5 blockade is observed, the lead molecule can be optimized to mitigate the hNav1.5 effect at an early stage during lead optimization with the guidance of in silico tools as exemplified in Section "In vivo Animal Models." Manual patch-clamp is used during the late pre-clinical phase to verify automated patch-clamp results and obtain more accurate drug concentration data. During clinical candidate selection, non-rodent in vivo telemetry studies, typically jacketed dogs and/or monkeys in ascending dose general toxicity studies, are used to detect potential sodium channel effects in vivo. Additional pre-clinical assays such as isolated heart models may be used as needed on a case-by-case basis at any stage, including after the compound is tested in man (reverse translation). During the Investigational New Drug (IND) enabling phase ECGs are recorded in non-rodent cardiovascular single dose safety pharmacology studies with implanted electrodes and in repeat dose toxicity studies with either jackets or external electrodes. At this stage of development, we consider hNav1.5 inhibition as a safety signal, whether detected in vitro through patch-clamp assay or in an isolated heart model, or in vivo using non-rodent telemetry models. It is worthwhile to stress that a risk benefit analysis and an integrated risk assessment must be performed for each individual molecule. The assessment should address exposureresponse relationships for the compound's unwanted effect on depolarization/conduction compared with the exposure for its primary pharmacodynamic and estimated therapeutic effects. Special consideration should also be given to the anticipated target population, especially patients with pre-existing cardiac risk factors (e.g., structural heart disease, prior myocardial infarction, etc.) or known cardiac sodium channel gene variants.

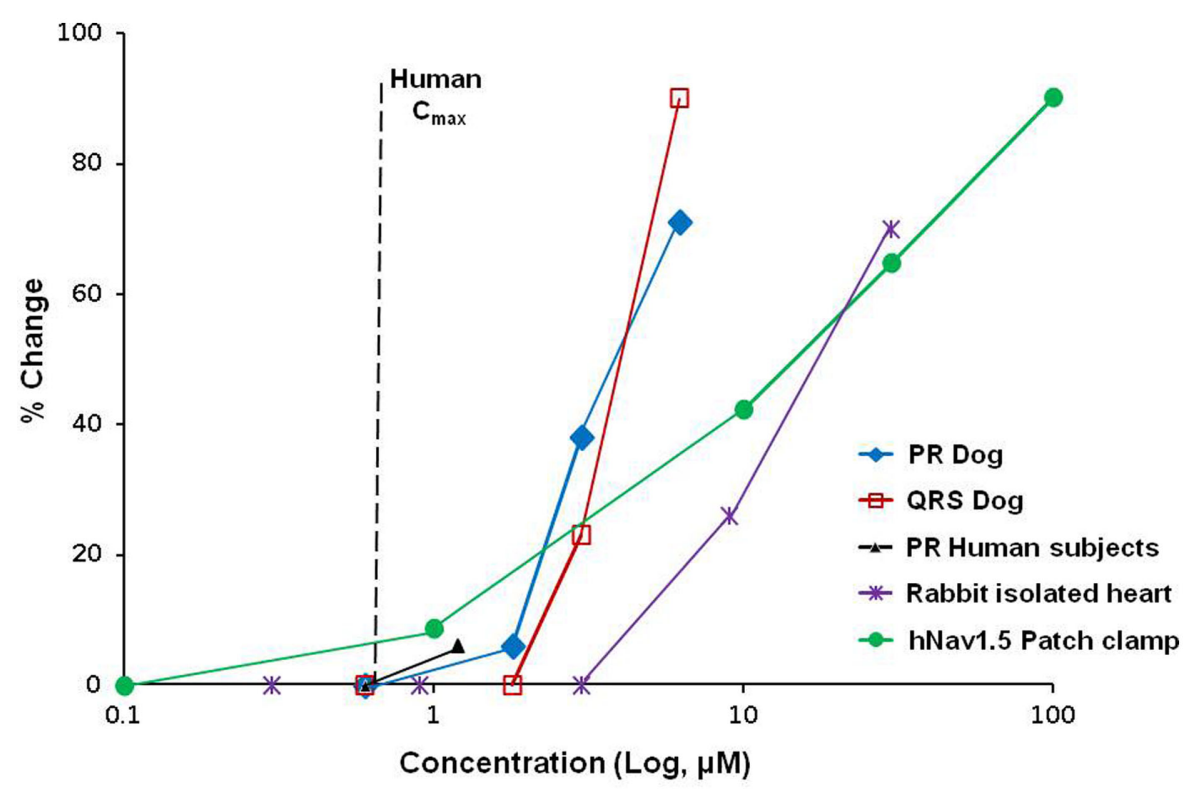

FIGURE 2 | Pre-clinical to clinical translation of sodium channel inhibition. Overlay plots of NVP-4 concentration-response curves are shown for effects on PR interval in human subjects, QRS and PR intervals in dogs, conductance in isolated rabbit hearts, and hNav1.5 current amplitude in manual patch-clamp. In vivo dog and human data are plotted as maximum free plasma concentration $\left(C_{\max }\right)$. Plasma protein binding of NVP-4 in humans and dogs is 77 and $85 \%$, respectively. Vertical dashed line indicates human free $C_{\max }$ at efficacious dose $(0.67 \mu \mathrm{M})$. 


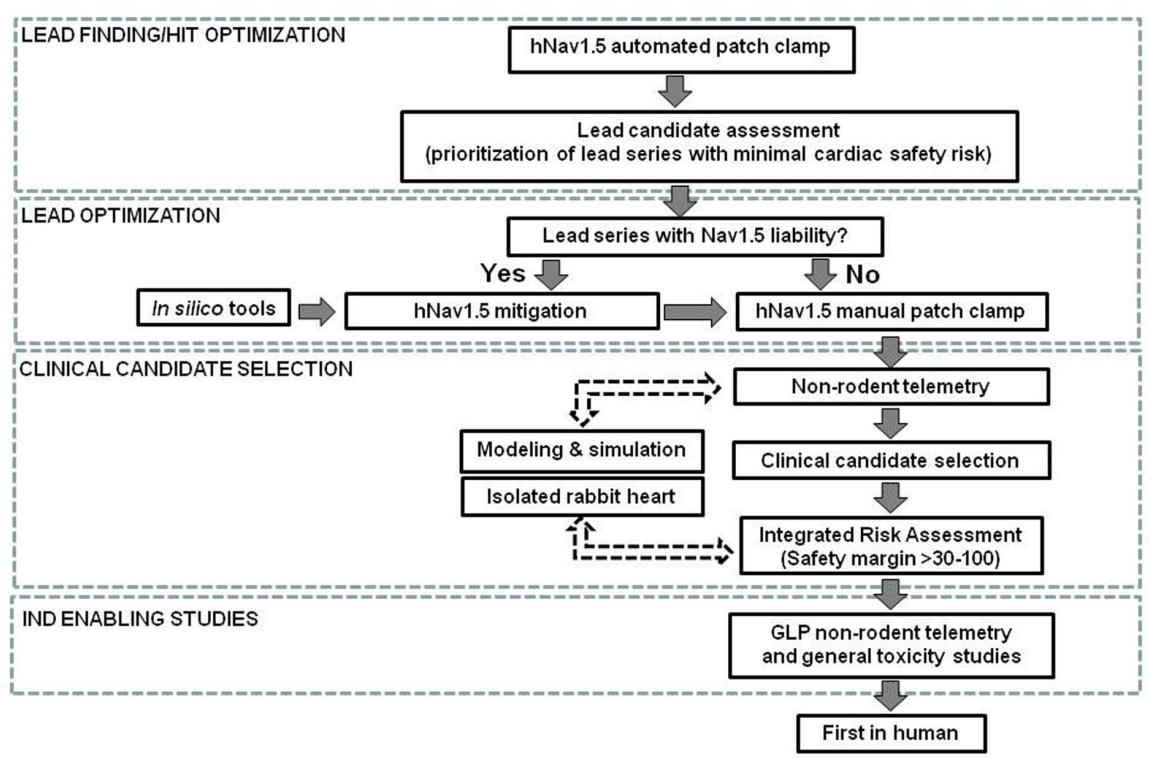

FIGURE 3 | Cardiac sodium channel safety assessment schematic. Non-rodent telemetry includes dog or non-human primates. Safety margins are calculated by dividing $h N a v 1.5 I C_{50}$, lowest effect concentration in the rabbit heart or free $C_{\max }$ for lowest efficacious dose in non-rodent telemetry by free $C_{\max }$ at estimated efficacious plasma concentration in human. IND, Investigational New Drug; GLP, Good Laboratory Practice. Dotted lines indicate case-by-case use (see text for details).

\section{TRANSLATIONAL VALUE OF THE PRE-CLINICAL ASSESSMENT AND CLINICAL PERSPECTIVE}

As is true for many other cases in drug development, translating pre-clinical information regarding hNav1.5 into clinically meaningful predictions is challenging. The experience with sodium channel blocking drugs in the CAST trial taught us that suppression of ventricular premature depolarizations via hNav1.5 blockade was an unsafe and inappropriate therapeutic goal in patients who had suffered a myocardial infarction. So, the question remains: what is an appropriate surrogate to indicate a clinical safety risk based on hNav1.5 inhibition?

Two commonly used surrogate markers are the PR and QRS intervals on the ECG. The PR interval represents AV conduction time, and can be prolonged by hNav1.5 or hCav1.2 blockers. However, many other factors affect this interval including heart rate and autonomic tone. In addition, both specialized conduction tissues (sinoatrial node and AV node) and atrial myocytes contribute to the interval. Although these variables limit the sensitivity and specificity of the PR as a surrogate and its ultimate utility as a safety biomarker, it should not be ignored when prolongation is detected. The QRS width is also an easily quantified interval that is mechanistically linked to hNav1.5 blockade. Harmer et al. (2011) examined the relationship between hNav1.5 $\mathrm{IC}_{50}$ and QRS widening in humans and found that a QRS change can be evoked by a free plasma drug concentration 15-fold less than the corresponding IC $_{50}$. Cordes et al., 2009 came to a similar conclusion. The implication from these analyses is that a relatively small inhibition of hNav1.5, e.g., $<10 \%$, could be associated with QRS prolongation in man (Cordes et al., 2009).

What are the clinical implications of QRS widening via hNav1.5 blockade? The clinical manifestations will depend on specific factors such as on/off kinetics, use dependence, dosing regimen, and individual patient cardiac risk (e.g., those with cardiomyopathy). The true correlation between degree of QRS widening and hard clinical endpoints (e.g., arrhythmic death) requires largescale prospective trials of drugs with hNavl.5 blocking properties and QRS widening as the dose limiting toxicity. This situation would allow a risk/benefit assessment and lend insight into what degree of QRS widening might translate into pro-arrhythmia risk.

In the absence of this type of clinical validation for QRS as a surrogate marker for hNav1.5 mediated cardiac safety risk and based on their data, Harmer et al. (2011) have suggested that the pharmaceutical industry treat the relationship between hNav1.5 inhibition and QRS widening as we have the relationship between hERG inhibition and QT prolongation. That is, while the QT prolongation associated with hERG blockade is an imperfect surrogate for TdP and death, given a choice between a drug that prolongs QT and one that does not, the prudent choice would be to develop the latter. Similarly, given the potential for arrhythmic risk with QRS prolongation, the logical approach would be to remove or minimize hNav1.5 blocking activity in drug candidates before entering human trials. We agree with this position, particularly since clinical validation of QRS prolongation as a risk biomarker is not likely to be performed.

\section{LIMITATIONS OF THE STUDY}

This study is a work in progress and there are a number of limitations that should be acknowledged. In this review, we focus on electrophysiological effects of drug-induced hNav1.5 inhibition. The sodium channel inhibition may have various other cardiovascular effects such as impairment of cardiac contractility. However, these are not within the scope of this review and therefore are not 
covered. The data sets particularly the clinical data are limited. The $\mathrm{IC}_{50}$ for hNav1.5 inhibition is used to define the potency of the compounds as this measure is the most reliable part of the sigmoid concentration response (Redfern et al., 2003; Harmer et al., 2011). However, $\mathrm{IC}_{10}$ and/or $\mathrm{IC}_{20}$ values may be more accurate measures to translate in vitro potency into in vivo effects. Additionally, use of ratios, when calculating safety margins, mainly deal with the central tendency of populations and do not consider outliers. Finally, the data set presented in this work is limited to low molecular weight inhibitors of hNav1.5 and the recommendations may not apply to larger biologic molecules such as therapeutic proteins or monoclonal antibodies.

\section{SUMMARY AND RECOMMENDATIONS}

In contrast to the established meticulous standards required to characterize $\mathrm{I}_{\mathrm{Kr}}$ inhibition and QT prolongation risk during drug development, health authorities do not specifically request characterization of a drug candidate's effect on hNav1.5 and QRS complex. Thus, these studies are not routinely performed. However, there is ample evidence that hNav1.5 inhibition poses a significant cardiovascular risk.

Compounds that inhibit hNav1.5 and slow the rate of cardiac depolarization and conduction (manifested as QRS prolongation) can also cause life-threatening arrhythmias and sudden cardiac death, although the relationship between QRS widening and arrhythmias in healthy or diseased hearts is not well understood (Seger, 2006). Nonetheless, possible safety concerns regarding cardiac sodium channel inhibition in patients and the

\section{REFERENCES}

Abriel, H. (2007). Cardiac sodium channel Navl.5 and its associated proteins. Arch. Mal. Coeur Vaiss. 100, 787-793.

Adesanya, C. O., Yousuf, K. A., Co, C., Gaur, S., Ahmed, S., Pothoulakis, A., Suryaprasad, A., and Gupta, S. (2008). Is wider worse? QRS duration predicts cardiac mortality in patients with right bundle branch block. Ann. Noninvasive Electrocardiol. 13, 165-170.

Balser, J. R. (2001). The cardiac sodium channel: gating function and molecular pharmacology. J. Mol. Cell. Cardiol. 33, 599-613.

Bode-Schnurbus, L., Bocker, D., Block, M., Gradaus, R., Heinecke, A., Breithardt, G., and Borggrefe, M. (2003). QRS duration: a simple marker for predicting cardiac mortality in ICD patients with heart failure. Heart 89 , 1157-1162.

Bottino, D., Penland, R. C., Stamps, A., Traebert, M., Dumotier, B., Georgiva, A., Helmlinger, G., and Lett, G. S. (2006). Preclinical cardiac safety assessment of pharmaceutical compounds using an integrated systemsbased computer model of the heart. Prog. Biophys. Mol. Biol. 90, 414-443. Cao, X., Lee, Y. T., Holmqvist, M., Lin, Y., Ni, Y., Mikhailov, D., Zhang, H.,

central importance of cardiac adverse effects during the drug development process make it prudent to identify, eliminate or minimize this liability early in the drug development process. The potential for drug-induced inhibition of the cardiac sodium channel has increasingly gained attention in recent years (Chen et al., 2009; Harmer et al., 2011). Pharmaceutical companies and contract research laboratories have begun to employ broader screening against a panel of cardiac ion channels, including hNav1.5. Recent data from Harmer et al. (2011) showed a good correlation between $\mathrm{IC}_{50}$ values for cardiac sodium channel inhibition in an automated electrophysiology-based assay and potency for QSR widening in humans for a wide range of compounds. The Novartis approach starts with in vitro assays for hNav1.5 and expands the safety assessment to integrate the judicious use of in silico, in vitro, and in vivo tools. Early data from implementation of this approach show promising results although the translational experience is still limited. We believe that a systematic approach incorporating in vitro assays, in vivo models and in silico modeling can provide a better understanding of the effects of cardiac sodium channel and enable better decision making in early drug development.

\section{ACKNOWLEDGMENTS}

We would like to thank Dr. William Dole and Dr. Laszlo Urban for their critical review of the manuscript, Dr. Berengere Dumotier and Dr. Yuichiro Adachi for the isolated rabbit heart data, Dr. Mats Holmqvist for Quattro hNav1.5 data, and Dr. Mark Deurinck for his contributions to in vivo data analysis.

Dunlop, J., Bowlby, M., Peri, R., Vasilyev, D., and Arias, R. (2008). Highthroughput electrophysiology: an emerging paradigm for ion-channel screening and physiology. Nat. Rev. Drug Discov. 7, 358-368.

Echt, D. S., Liebson, P. R., Mitchell, L. B., Peters, R. W., Obias-Manno, D., Barker, A. H., Arensberg, D., Baker, A., Friedman, L., and Greene, H. L. (1991). Mortality and morbidity in patients receiving encainide, flecainide, or placebo. The Cardiac Arrhythmia Suppression Trial. N. Engl. J. Med. 324, 781-788.

Freedman, R. A., and Steinberg, J. S. (1991). Selective prolongation of QRS late potentials by sodium channel blocking antiarrhythmic drugs: relation to slowing of ventricular tachycardia. Electrophysiologic study versus electrocardiographic monitoring trial (ESVEM) investigators. J. Am. Coll. Cardiol. 17, 1017-1025.

George, A. L. Jr., Varkony, T. A., Drabkin, H. A., Han, J., Knops, J. F., Finley, W. H., Brown, G. B., Ward, D. C., and Haas, M. (1995). Assignment of the human heart tetrodotoxin-resistant voltage-gated $\mathrm{Na}^{+}$channel alphasubunit gene (SCN5A) to band 3p21. Cytogenet. Cell Gene 68, 67-70.
Gintant, G. A., Gallacher, D. J., and Pugsley, M. K. (2011). The 'overlysensitive' heart: sodium channel block and QRS interval prolongation. Br. J. Pharmacol. 164, 254-259. Graf, B. M. (2001). The cardiotoxicity of local anesthetics: the place of ropivacaine. Curr. Top. Med. Chem. 1, 207-214.

Harmer, A., Valentin, J.-P., and Pollard, C. (2011). On the relationship between block of the cardiac $\mathrm{Na}^{+}$ channel and drug-induced prolongation of the QRS complex. Br. J. Pharmacol. 164, 260-273.

Harmer, A. R., Abi-Gerges, N., Easter, A., Woods, A., Lawrence, C. L., Small, B. G., Valentin, J. P., and Pollard, C. E. (2008). Optimisation and validation of a mediumthroughput electrophysiology-based hNav1.5 assay using IonWorks. J. Pharmacol. Toxicol. Methods 57 30-41.

Heath, B. M., Cui, Y., Worton, S., Lawton, B., Ward, G., Ballini, E., Doe, C. P., Ellis, C., Patel, B. A., and Mcmahon, N. C. (2011). Translation of flecainide- and mexiletine-induced cardiac sodium channel inhibition and ventricular conduction slowing from nonclinical models to clinical. J. Pharmacol. Toxicol. Methods 63, $258-268$. 
Heist, E. K., and Ruskin, J. N. (2010). Drug-induced arrhythmia. Circulation 122, 1426-1435.

Hodgkin, A. L., and Huxley, A. F. (1952). A quantitative description of membrane current and its application to conduction and excitation in nerve. J. Physiol. (Lond.) 117, 500-544.

Hondeghem, L. M. (1994). Computer aided development of antiarrhythmic agents with class IIIa properties. J. Cardiovasc. Electrophysiol. 5, 711-721.

Hondeghem, L. M., Lu, H. R., Van Rossem, K., and De Clerck, F. (2003). Detection of proarrhythmia in the female rabbit heart: blinded validation. J. Cardiovasc. Electrophysiol. 14, 287-294.

Hummel, S. L., Skorcz, S., and Koelling, T. M. (2009). Prolonged electrocardiogram QRS duration independently predicts long-term mortality in patients hospitalized for heart failure with preserved systolic function. J. Card. Fail. 15, 553-560.

Inoue, H., and Zipes, D. P. (1987). Changes in atrial and ventricular refractoriness and in atrioventricular nodal conduction produced by combinations of vagal and sympathetic stimulation that result in a constant spontaneous sinus cycle length. Circ. Res. 60, 942-951.

Ito, K., Nagafuchi, K., Taga, A., Yorikane, R., and Koike, H. (1996). Possible involvement of altered $\mathrm{Na}^{+}$. $\mathrm{Ca}^{2+}$ exchange in negative inotropic effects of class I antiarrhythmic drugs on rabbit and rat ventricles. J. Cardiovasc. Pharmacol. 27, 355-361.

Jamieson, C., Moir, E. M., Rankovic, Z., and Wishart, G. (2006). Medicinal chemistry of hERG optimizations: highlights and hang-ups. J. Med. Chem. 49, 5029-5046.

Keating, M. T., and Sanguinetti, M. C. (2001). Molecular and cellular mechanisms of cardiac arrhythmias. Cell 104, 569-580.

Larbig, R., Torres, N., Bridge, J. H., Goldhaber, J. I., and Philipson, K. D. (2010). Activation of reverse $\mathrm{Na}^{+}$$\mathrm{Ca}^{2+}$ exchange by the $\mathrm{Na}^{+}$current augments the cardiac $\mathrm{Ca}^{2+}$ transient: evidence from NCX knockout mice. J. Physiol. (Lond.) 588, 3267-3276.

Lu, H. R., Rohrbacher, J., Vlaminckx, E., Van Ammel, K., Yan, G. X., and Gallacher, D. J. (2010). Predicting drug-induced slowing of conduction and pro-arrhythmia: identifying the 'bad' sodium current blockers. Br. J. Pharmacol. 160, 60-76.

Lu, H. R., Vlaminckx, E., Van De Water, A., Rohrbacher, J., Hermans, A. and Gallacher, D. J. (2007) In-vitro experimental models for the risk assessment of antibioticinduced QT prolongation. Eur. J. Pharmacol. 577, 222-232.

Moreno, J. D., Zhu, Z. I., Yang, P.-C., Bankston, J. R., Jeng, M.-T., Kang, C., Wang, L., Bayer, J. D., Christini, D. J., Trayanova, N. A., Ripplinger, C. M., Kass, R. S., and Clancy, C. E. (2011). A computational model to predict the effects of class I anti-arrhythmic drugs on ventricular rhythms. Sci. Transl. Med. 3, 98ra83.

Noble, D., and Rudy, Y. (2001). Models of cardiac ventricular action potentials: iterative interaction between experiment and simulation. Philos. Trans. R. Soc. Lond. A 359, 1127-1142.

Papadatos, G., Alkarouri, M., Gillet, V. J., Willett, P., Kadirkamanathan, V., Luscombe, C. N., Bravi, G., Richmond, N. J., Pickett, S. D., Hussain, J., Pritchard, J. M., Cooper, A. W. J., and Macdonald, S. J. F. (2010). Lead optimization using matched molecular pairs: inclusion of contextual information for enhanced prediction of hERG inhibition, solubility, and lipophilicity. J. Chem. Inf. Model. 50, 1872-1886.

Piccini, J. P., Whellan, D. J., Berridge, B. R., Finkle, J. K., Pettit, S. D., Stockbridge, N., Valentin, J.-P., Vargas, H. M., Krucoff, M. W., and Group, C. H. W. (2009). Current challenges in the evaluation of cardiac safety during drug development: translational medicine meets the critical path initiative. Am. Heart J. 158, 317-326.

Pressler, M. L., Warner, M. R., Rubart, M., Rardon, D. P. and Zipes, D. P. (1995). In vivo and in vitro electrophysiologic effects of terodiline on dog myocardium. J. Cardiovasc. Electrophysiol. 6, 443-454.

Redfern, W. S., Carlsson, L., Davis, A. S., Lynch, W. G., Mackenzie, I., Palethorpe, S., Siegl, P. K., Strang, I., Sullivan, A. T., Wallis, R., Camm, A. J., and Hammond, T. G. (2003). Relationships between preclinical cardiac electrophysiology, clinical
QT interval prolongation and torsade de pointes for a broad range of drugs: evidence for a provisional safety margin in drug development. Cardiovasc. Res. 58, 32-45.

Rook, M. B., Evers, M. M., Vos, M. A., and Bierhuizen, M. F. (2012). Biology of cardiac sodium channel Nav1.5 expression. Cardiovasc. Res. 93, 12-23.

Ruan, Y., Denegri, M., Liu, N., Bachetti, T., Seregni, M., Morotti, S., Severi, S., Napolitano, C., and Priori, S. G. (2010). Trafficking defects and gating abnormalities of a novel SCN5A mutation question genespecific therapy in long QT syndrome type 3. Circ. Res. 106, 1374-1383.

Seger, D. L. (2006). A critical reconsideration of the clinical effects and treatment recommendations for sodium channel blocking drug cardiotoxicity. Toxicol. Rev. 25, 283-296.

Starmer, C. F., Colatsky, T. J., and Grant, A. O. (2003a). What happens when cardiac Na channels lose their function? 1. Numerical studies of the vulnerable period in tissue expressing mutant channels. Cardiovasc. Res. 57, 82-91.

Starmer, C. F., Grant, A. O., and Colatsky, T. J. (2003b). What happens when cardiac Na channel function is compromised? 2. Numerical studies of the vulnerable period in tissue altered by drugs. Cardiovasc. Res. 57, 1062-1071.

Starmer, C. F., Lancaster, A. R., Lastra, A. A., and Grant, A. O. (1992). Cardiac instability amplified by usedependent $\mathrm{Na}$ channel blockade. Am. J. Physiol. 262, H1305-H1310.

Starmer, C. F., Lastra, A. A., Nesterenko, V. V., and Grant, A. O. (1991). Proarrhythmic response to sodium channel blockade. Theoretical model and numerical experiments. Circulation 84, 1364-1377.

Sumner, G., Salehian, O., Yi, Q., Healey, J., Mathew, J., Al-Merri, K., AlNemer, K., Mann, J. F. E., Dagenais, G., and Lonn, E. (2009). The prognostic significance of bundle branch block in high-risk chronic stable vascular disease patients: a report from the HOPE trial. J. Cardiovasc. Electrophysiol. 20, 781-787.

Takahara, A., Dohmoto, H., Yoshimoto, R., Sugiyama, A., and Hashimoto, K. (2001). Cardiovascular action of a cardioselective $\mathrm{Ca}^{2+}$ channel blocker AH-1058 in conscious dogs assessed by telemetry. Eur. J. Pharmacol. 413, 101-108.

Tfelt-Hansen, J., Winkel, B., Grunnet, M., and Jespersen, T. (2010). Inherited cardiac diseases caused by mutations in the Nav1.5 sodium channel. J. Cardiovasc. Electrophysiol. 21, 107-115.

Thanacoody, H. K., and Thomas, S. H. (2005). Tricyclic antidepressant poisoning: cardiovascular toxicity. Toxicol. Rev. 24, 205-214.

Van Noord, C., Eijgelsheim, M., and Stricker, B. H. C. (2010). Drugand non-drug-associated QT interval prolongation. Br. J. Clin. Pharmacol. 70, 16-23.

Vaughan Williams, E. M. (1992). The relevance of cellular to clinical electrophysiology in classifying antiarrhythmic actions. J. Cardiovasc. Pharmacol. 20(Suppl. 2), S1-S7.

Vieweg, W. V., and Wood, M. A. (2004). Tricyclic antidepressants, QT interval prolongation, and torsade de pointes. Psychosomatics 45, 371-377.

Warner, B., and Hoffmann, P. (2002). Investigation of the potential of clozapine to cause torsade de pointes. Adverse Drug React. Toxicol. Rev. 21, 189-203.

Conflict of Interest Statement: All authors are employees of Novartis Institutes for Biomedical Research Inc.

Received: 19 December 2011; accepted: 12 January 2012; published online: 26 January 2012.

Citation: Erdemli G, Kim AM, Ju H, Springer $C$, Penland $R C$ and Hoffmann PK (2012) Cardiac safety implications of hNav1.5 blockade and a framework for pre-clinical evaluation. Front. Pharmacol. 3:6. doi: 10.3389/fphar.2012.00006

This article was submitted to Frontiers in Pharmaceutical Medicine and Outcomes Research, a specialty of Frontiers in Pharmacology.

Copyright (C) 2012 Erdemli, Kim, Ju, Springer, Penland and Hoffmann. This is an open-access article distributed under the terms of the Creative Commons Attribution Non Commercial License, which permits non-commercial use, distribution, and reproduction in other forums, provided the original authors and source are credited. 